\title{
The spatial Ultimatum game revisited
}

\author{
Jaime Iranzo ${ }^{\mathrm{a}, \mathrm{b}}$, Javier Román ${ }^{\mathrm{c}}$, Angel Sánchez ${ }^{\mathrm{b}, \mathrm{c}, \mathrm{d}, *}$ \\ a Centro de Astrobiología CSIC-INTA, 28850 Torrejón de Ardoz, Madrid, Spain \\ ${ }^{\mathrm{b}}$ Instituto de Biocomputación y Física de Sistemas Complejos (BIFI), Universidad de Zaragoza, Campus Río Ebro, 50018 Zaragoza, Spain \\ c Grupo Interdisciplinar de Sistemas Complejos (GISC), Departamento de Matemáticas, Universidad Carlos III de Madrid, \\ Avenida de la Universidad 30, 28911 Leganés, Madrid, Spain \\ d Instituto de Ciencias Matemáticas CSIC-UAM-UC3M-UCM, c/ Nicolás Cabrera 14-16, 28049 Cantoblanco, Madrid, Spain
}

\section{A R T I C L E I N F O}

\section{Article history:}

Received 22 October 2010

Received in revised form

19 February 2011

Accepted 23 February 2011

Available online 1 March 2011

Keywords:

Evolution

Fairness

Rationality

Game theory

Spatial dynamics

\begin{abstract}
A B S T R A C T
We revisit the issue of the emergence of fair behavior in the framework of the spatial Ultimatum game, adding many important results and insights to the pioneering work by Page et al. [2000. The spatial Ultimatum game. Proc. R. Soc. London B 267, 2177], who showed in a specific example that on a two dimensional setup evolution may lead to strategies with some degree of fairness. Within this spatial framework, we carry out a thorough simulation study and show that the emergence of altruism is a very generic phenomenon whose details depend on the dynamics considered. A very frequent feature is the spontaneous emergence and fixation of quasiempathetic individuals, whose offers are very close to their acceptance thresholds. We present analytical arguments that allow an understanding of our results and give insights on the manner in which local effects in evolution may lead to such non rational or apparently maladaptive behaviors.
\end{abstract}

\section{Introduction}

The evolution of cooperation and the difference between humans and other primates are two intimately related questions that attract a lot of interest among researchers in fields from biology through economics to psychology. From a biological viewpoint, understanding the emergence of cooperation is crucial to unveil the mechanisms of the evolution of complex organisms through the association of simpler entities (Maynard Smith and Szathmary, 1995). On the other hand, the question is socio economically relevant because human societies have arisen mostly due to the readiness of humans to cooperate with non kin even when the interaction is unlikely to take place again in the future, or in sizeable groups (Fehr and Fischbacher, 2003). This type of behavior has been pointed out as one of the charac teristics that set humans apart from all other animals (Kappeler and van Schaik, 2006). Today, this topic remains a very active field, and different mechanisms are being proposed to understand the evolutionary origin of cooperation (Nowak, 2006).

At the roots of many cooperative behaviors in humans lies altruism (Gintis, 2000). Altruism is defined as the capacity to perform costly acts that confer benefits on others and, hence, it is

\footnotetext{
* Corresponding author at: Grupo Interdisciplinar de Sistemas Complejos (GISC), Departamento de Matemáticas, Universidad Carlos III de Madrid, Avenida de la Universidad 30, 28911 Leganés, Madrid, Spain.

E-mail address: anxo@math.uc3m.es (A. Sánchez).
}

in principle detrimental for the altruistic individuals. In fact, seemingly altruistic acts can be understood, when looked at in depth, in terms of kin selection (Hamilton, 1964) or reciprocity (Trivers, 1971; Axelrod and Hamilton, 1981), for instance. These interpretations notwithstanding, there are many reports of truly altruistic behavior in humans, in particular in experiments among anonymous subjects that interact only once (see, e.g., Kagel and Roth, 1995; Camerer, 2003; Fehr and Fischbacher, 2003; Henrich et al., 2004 for reviews).

A framework that has proven itself particularly suited to explore this issue is the Ultimatum game (Güth et al., 1982). The Ultimatum game consists of two players deciding how to distribute a reward. One of the players, known as proposer, makes an offer and the other player (the responder) decides whether to accept it or not. There is only one opportunity to make a deal: If the responder accepts, the reward is distributed between the players according to the proposer's offer, otherwise both players receive nothing. Rational players's behavior is easy to predict: Given that any positive amount should be acceptable for the responder, the proposer will offer such minimum quantity, which will be accepted. On the contrary, a wealth of experimental evidence about the Ultimatum game (Camerer, 2003; Henrich et al., 2004) has established clearly that humans are universally altruistic, in so far as subjects from ethnic groups all over the world show some degree of fairness: Indeed, most proposers offer a fair share, mean offers spanning the range from 25 to 57 percent of the amount to be splitted. Interestingly, it is also observed 
often that unfair offers (typically below 20 percent) are rejected many times, although there is larger variability in this respect than in the proposers' behavior.

A great deal of research has been devoted to understand the origins of this behavior. One of the factors that has been proposed to play an important role in this issue is culture (see, e.g., Gintis, 2003; Hammerstein, 2003; Oosterbeek et al., 2004; Henrich et al., 2004; Marlowe et al., 2007) but other works point out the need to consider the influence of genetic or biological features (Wallace et al., 2007; Burnham, 2007). Furthermore, instances of fair behavior related to those arising on the Ultimatum game have been reported among non human primates (Brosnan and de Waal, 2003; Kappeler and van Schaik, 2006) (note, however, that rational like behavior has also been found in chimpanzees (Jensen et al., 2007)). In fact, the Ultimatum game has indeed very direct biological applications beyond the context of human behavior. This is the case, e.g., of the division of parental care between the sexes, when mate desertion has a positive effect on the deserting individual's reproductive success (Hammerstein, 2006). Another situation with Ultimatum game characteristics arises when indi viduals need to divide a reward or a prey, such as in cooperative hunting. Thus, among chimpanzees dominant males, who did not necessarily participate in the hunt itself, tend to end up with the prey and control its distribution (Boesch et al., 2006). Even outside of the realm of primates, sharing rewards or food may lead to contexts related to the Ultimatum game, such as when fish distribute among patches to feed (Milinski, 1984). Therefore, even if culture may have contributed to the appearance of altruistic behaviors in humans, these are likely to have complex causes, in which more biological considerations should enter as well, and more so if we think of non human animals.

In view of the above comments, explanations of non rational actions in the Ultimatum game in evolutionary terms are very relevant. Several such mechanisms have been proposed in the past few years. Thus, it has been argued that if selection is rapid, i.e., if reproduction takes place at a faster scale than interaction among individuals, altruistic behavior may be evolutionary selected (Sánchez and Cuesta, 2005). Another possible scenario leading to large offers in the Ultimatum game is the existence of empathy (Page and Nowak, 2000, 2002), in which players choose as their offer the smallest amount they are willing to accept. Finally, elaborating on ideas proposed by Nowak and May for Prisoner's Dilemma (Nowak and May, 1992), it was suggested that the existence of a spatial structure could support the appearance of altruistic behavior. The original proposal was done by Page et al. (2000), who carried out a comparative study of the Ultimatum game on a well mixed population, a one dimensional system (a ring), and a sketchy analysis of two dimensional lattices. They concluded that large offer levels, around a 34\% of the amount to share, emerged and were stable in the ring and the lattice, while evolution converged to the rational solution on a well mixed population. A similar result was later shown for a generalization of the Ultimatum game to describe collaborations by Killingback and Studer (2001). More recently, the effect of complex (non spatial) networks of interactions was addressed by Kuperman and Risau Gusman (2008), Eguíluz and Tessone (2009), and Sinatra et al. (2009), finding again that large offers appeared in the population.

In this paper we revisit the issue of the effect of the spatial structure on the Ultimatum game by focusing on the two dimensional case. We largely expand the only previous work on the subject (Page et al., 2000) by considering different dynamical rules (possibly with noise), including the possibility of empathetic players, and presenting detailed analytical arguments that allow for understanding the mechanisms at work in the evolution of the strategies.

\section{Model}

We consider a set of agents playing the spatial Ultimatum game, i.e., they are located on the nodes of two dimensional square lattices and play the game only with their neighbors. Most results have been obtained for connectivity equal to 4 (players connected to their neighbors north, south, east and west) and size $40 \times 40$. For comparison, some other simulations have been performed with network size $10 \times 10$ or larger connectivities. We will mention on this different parameters as needed along the paper.

Without loss of generality, the total reward will be hereafter normalized to the unity. The strategy of every player is then characterized by two parameters, $p, q \in[0,1]$. The value of $p$ indicates the fraction of the reward offered by the player when acting as proposer. On the other side, $q$ denotes the acceptance threshold, i.e., the minimum quantity that the player accepts when acting as responder. We will study two different possibi lities for this two parameters: a empathetic setting, in which players offer the minimum amount they are ready to accept, so $p=q$ and strategies are defined by a single parameter, and an independent setting in which both parameters evolve indepen dently. With this notation, an interaction between players $i$ and $j$, with $i$ taking the role of proposer and $j$ the responder, takes place as follows $\left(\Delta \Pi_{i j}^{O}\right.$ and $\Delta \Pi_{j i}^{R}$ denote the increment of payoff for $i$ and $j$, respectively):

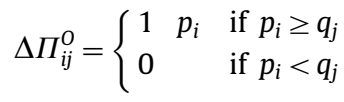

$\Delta \Pi_{j i}^{R}= \begin{cases}p_{i} & \text { if } p_{i} \geq q_{j} \\ 0 & \text { if } p_{i}<q_{j}\end{cases}$

Every time step, the lattice is swept and every player partici pates in an Ultimatum game with each of her neighbors. Regard ing the assignation of roles (proposer and responder) between the players, two settings have been considered: non random $(n R)$, in which the focal agent plays as proposer (and therefore she plays as responder when her neighbors are the focal agents), and random $(R)$, in which roles are assigned randomly in every encounter, so that the same individual can act twice as proposer or responder. In the latter case, the expectation for the payoff increment of $i$ after a double interaction with $j$ is

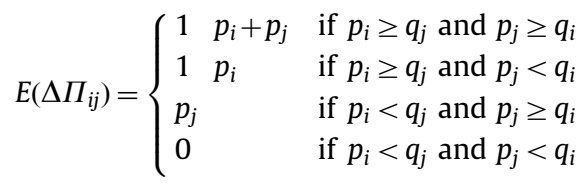

In case non random roles are assigned, the previous expectation becomes the exact payoff after a double $(i, j)$ interaction. The final payoff is the sum of the increments after playing with all neighbors.

Subsequently to the games, players update their strategies. Each individual compares its final payoff with that of its neigh bors and modify its parameters $\left(p_{i}, q_{i}\right)$ according to one of the following two update rules. The first one, unconditional imitation (UI), also known as "imitation of the best", stipulates that each player copies the strategy of the neighbor with highest payoff, provided it is greater than the player's. As a result, this is a deterministic rule. This update procedure was first proposed by Nowak and May (1992) and has been widely used in the literature since then. The second rule, proportional imitation, is defined by having each player $i$ select one neighbor $j$ at random. Then, provided that the neighbor's payoff is greater, her strategy 
Table 1

Summary of the variations of the model considered in this study.

\begin{tabular}{lll}
\hline Alternative & Possible settings & Abbrev. \\
\hline Parameters $p, q$ & Empathetic/independent & $p \quad q / p, q$ \\
Role assignment & Random/once prop. - once resp. & $R / n R$ \\
Updating rule & Unconditional/proportional imitation & $U I / P I$ \\
Noise in the copy & With/without noise & $N / n N$ \\
\hline
\end{tabular}

will be copied with probability

$\mathcal{P}_{i j}=\frac{\Pi_{j} \Pi_{i}}{2 \max \left\{k_{i}, k_{j}\right\}}$

where $k_{i}$ and $k_{j}$ are the number of neighbors of $i$ and $j$ respectively. The denominator takes this form in order to ensure that $\mathcal{P}_{i j} \leq 1$. This update rule is also known as "replicator rule", since in the thermodynamical limit it results in the replicator equation, as shown by Helbing (1992) and Schlag (1998). Again, this rule is a common choice in research on evolutionary games. In addition, to address the effect of noise or mistakes, we have considered two scenarios, with or without noise in the copy of parameters (noise and no noise, abbreviated as $N$ and $n N$ ). Noise has been implemented by adding to the copied parameters a random value uniformly distributed in [ $0.001,0.001]$ (not necessarily the same for $p$ and $q$ ). The choice of the above rules is due to the fact that they have not been considered in the literature about the spatial Ultimatum game. Indeed, Page et al. used a Moran like process (Moran, 1962; Roca et al., 2009b) in which all neighbors try to take over the focal one and succeed with probability proportional to their payoffs. There fore, it is important to consider additional update rules that can add to our knowledge on this problem as it has been shown that in $2 \times 2$ games, they can lead to very different outcomes of the evolution (Roca et al., 2009a,b).

The final stage of a time step is that all payoffs are reset to zero. Therefore, there is neither memory nor information about neighbors strategies in the model here presented. Simulations were run for a maximum of $4 \times 10^{4}$ generations, stopping before if equilibrium was reached. A typical outcome of single simulations was the convergence of all the population to the same strategy, with all $p$ and $q$ values located within a narrow interval corre sponding to the magnitude of noise. In order to obtain frequency distributions for that final strategy averages were taken for $10^{4}$ independent realizations. A summary of the different conditions explored in this work is given in Table 1.

\section{Results: empathetic players}

As stated above, this setting is characterized by the fixed relationship $p=q$. On a well mixed population, the constraint that $p=q$ leads to offers close to one half of the total reward $(p=1 / 2)$ (Page and Nowak, 2002; Sánchez and Cuesta, 2005). In other words, empathy promotes fair behaviors. Our aim here is to contrast this result when players interact no longer with all the population but with a small, highly structured neighborhood.

The main result of our simulation is that for almost all the variations of our model, the population converges towards the well mixed population behavior, that is $p=1 / 2$ (Fig. 1b). In particular, this is the case as soon as any source of noise is introduced, either through random role assignment, proportional imitation or noise in the copy of parameters. Due to finite size effects, the final value in small populations (size $10 \times 10$ ) can be rather smaller than $1 / 2$ if no noise is introduced, as will be explained below. Nevertheless, the convergence of the population to a unique offer is a general feature. The only exception to the above outcome takes place for the setting

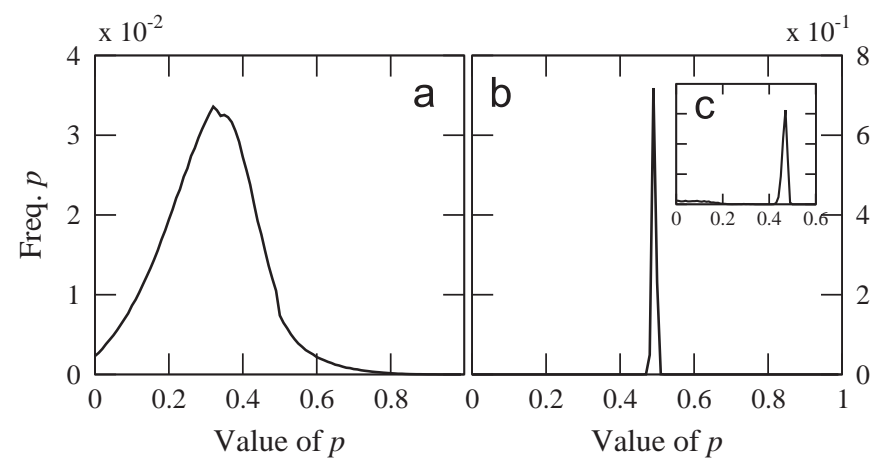

Fig. 1. Observed outcomes with empathetic players $\left(\begin{array}{ll}p & q\end{array}\right)$. Plots show the final distribution of offers averaged over $10^{4}$ realizations. Two major outcomes are possible: (a) stable coexistence of several offers in the population - only for the deterministic setting - and (b) mean-field behavior, with $p \approx 1 / 2$. Inset (c) shows a peculiar behavior that appears in large networks with setting $\{U I / R / n N\}$ and is characterized by fixation of low $p$ strategies in some realizations.

$\{U I / n R / n N\}$, when there is no source of stochasticity in evolution. In this case, many different values of parameter $p$ coexist in the population in a stable way (Fig. 1a). From a spatial point of view, stable clusters of individuals with the same $p$ are formed that can neither invade nor be invaded by their neighbors. Thus, coexistence is a result of the spatial structure of the population: In fact, we checked that if the UG takes place on random networks (even with homogeneous degree), the coexistence of offers is lost.

In order to understand why random roles, proportional imitation and noise prevent coexistence and lead to the well mixed result, we will follow Page et al. (2000) and consider a $3 \times 3$ cluster (or, alternatively, cross like configurations) of individuals with a differ ent strategy than that of the surrounding population. By comparing the payoff of every player it is possible to predict (exactly in the deterministic setting and approximately in the stochastic ones) what will happen to the cluster. Let $p_{\text {pop }}$ be the offer parameter of the population and $p_{\mathrm{cl}}$ that of the players in the cluster. Let us denote by $a, b, c, d$ the players involved in the interaction cluster environment, with $d$ at the corner of the cluster, $c$ at the lateral of it and $a, b$ outside the cluster in contact with $c$ and $d$, respectively, as shown in Fig. 2. We will consider several cases separately in what follows, in order to assess the effects of the different types of stochasticity we are studying.

\subsection{Cluster analysis: deterministic case $\{U I / n R / n N\}$}

We will begin by analyzing the deterministic situation, i.e., when there are no sources of noise in the model. First, consider a cluster of players whose $p$ is further from $1 / 2$ than the surround ing population, $p_{\mathrm{cl}}<p_{\mathrm{pop}} \leq 1 / 2$. When an individual plays with other of the same type two deals are made (as proposer and responder) and both players get payoff equal to 1 . However, since $p_{\mathrm{cl}}<q_{1}$, distinct players at the interface only accept the deal when the player in the cluster acts as responder. According to Eq. (3), the payoffs for every player are (see Fig. 2a)

$\Pi_{a}=\Pi_{b}=3+\left(1 \quad p_{\mathrm{pop}}\right) \geq 3.5$

$\Pi_{c}=3+p_{\text {pop }} \leq 3.5$

$\Pi_{d}=2+2 p_{\text {pop }} \leq 3$

Note that the individual at the center of the cluster, as well as those inside the surrounding population get a total payoff equal to 4. Evolution by unconditional imitation consists of looking for the neighbor with greatest payoff and imitating it. For players $a$ and $b$ the richest neighbor is that with payoff 4 inside the 


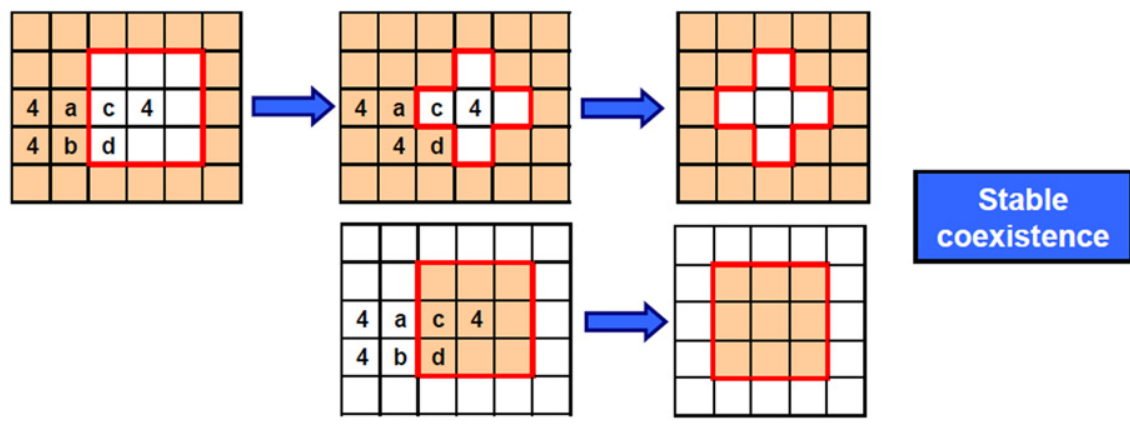

b

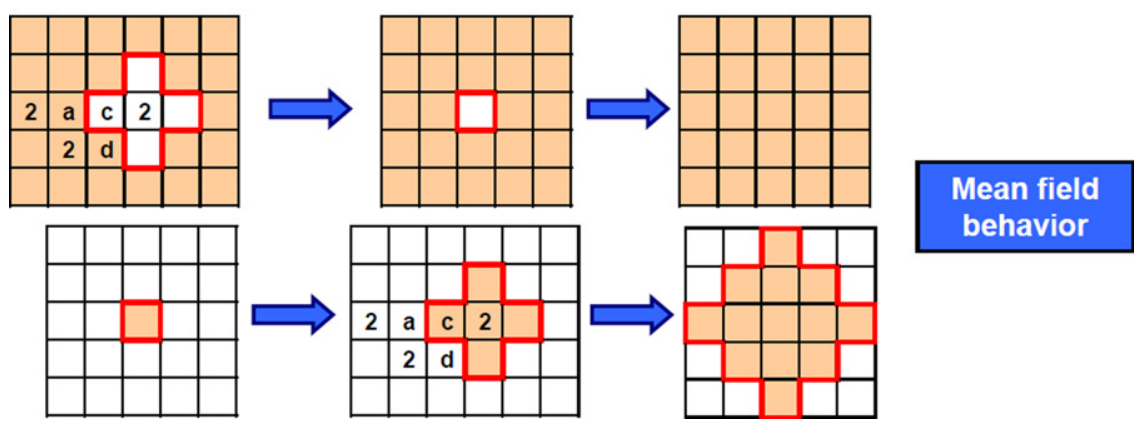

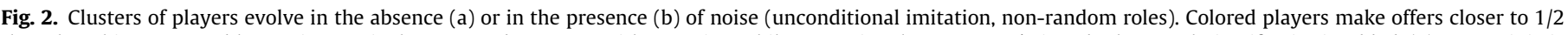

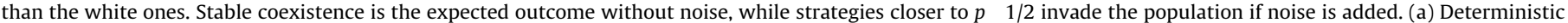
setting $\{\mathrm{UI} / \mathrm{nR} / \mathrm{nN}\}$, (b) Noise $\{\mathrm{UI} / \mathrm{nR} / \mathrm{N}\}$. (For interpretation of the references to color in this figure legend, the reader is referred to the web version of this article.)

population, so their strategy will remain the same. Player $c$ is in contact with the center of the cluster, whose payoff is equal to 4 , so it will not change either. Finally, the neighbors of player $d$ are $b$ (richer) and $c$ : Imitation of $b$ will change the strategy of $d$ to the majority one. As a result, a $3 \times 3$ cluster with $p_{\mathrm{cl}}<p_{\text {pop }}$ becomes a cross shaped structure. In the cross cluster, all individuals are connected to the central one, who still has payoff equal to 4 . Therefore, the resulting cross cluster is stable.

Always within the deterministic dynamics, consider now the case when individuals in the cluster have greater $p$ than the surrounding population, $p_{\text {pop }}<p_{\mathrm{cl}} \leq 1 / 2$. Now the payoffs become

$\Pi_{a}=\Pi_{b}=3+p_{\mathrm{cl}} \leq 3.5$

$\Pi_{c}=3+\left(1 \quad p_{\mathrm{cl}}\right) \geq 3.5$

$\Pi_{d}=2+2\left(1 \quad p_{\mathrm{cl}}\right) \geq 3$

Players $a, b$ and $c$ are in contact with individuals of their same type with payoff 4 , so their strategy will not change. Player $d$ best neighbor is $c$, that also belongs to the cluster. Hence, the $3 \times 3$ is a stable configuration. In the same way, it can be shown that a cross structure is also stable. The conclusion of this analysis is that small clusters can neither invade nor be invaded, no matter if their $p$ is greater or lower than that of their surroundings. In consequence, coexistence is the expected outcome in the deterministic setting.

\subsection{Cluster analysis: unconditional imitation with noise $\{\mathrm{UI} / n R / N\}$}

We can now turn to the discussion of the effect of noise. When there is noise in the system, there will be no more individuals with exactly the same value of $p$ (note that $q$ is fixed for every player equal to his $p$ ). Even if an individual plays with other of the same type, their parameters will still be slightly different and as a result only one deal will be finalized (that in which the player with greater $p$ acts as proposer). On average, when a player interacts with others of the same type half of the deals will be made as proposer and half as responder; hence, the expected payoff will be $p_{i} / 2+\left(\begin{array}{ll}1 & p_{i}\end{array}\right) / 2=1 / 2$. This result is in contrast with the case without noise, where players of the same type received a payoff of 1 . This is the main difference between both settings and has strong consequences in the evolution of offers.

In order to apply the same cluster analysis as before, we will approximate the payoff between players of the same type by means of the above expected value. This will give an idea of what will occur on average. Now the total payoff in the center of the cluster, as well as inside the population, is 2 . As before, let us begin with lower offer than the surroundings, $p_{\mathrm{cl}}<p_{\mathrm{pop}} \leq 1 / 2$. In the absence of noise, the stable configuration for that cluster is the cross structure. Let it be the starting shape of the cluster, the payoffs for every player are (see Fig. 2b)

$$
\begin{aligned}
& \Pi_{a}=3 / 2+\left(1 \quad p_{\mathrm{pop}}\right) \geq 2 \\
& \Pi_{c}=1 / 2+3 p_{\mathrm{pop}} \leq 2 \\
& \Pi_{d}=1+2\left(1 \quad p_{\mathrm{pop}}\right) \geq 2
\end{aligned}
$$

According to these payoffs, player $c$ will imitate $a$ or $d$ and only the central individual in the cluster will resist. This remaining individual will disappear in the next generation. Results are the same if a square cluster is considered.

Now suppose a single individual with parameter $p_{\mathrm{cl}}$ greater than the rest of the population $\left(p_{\text {pop }}<p_{\mathrm{cl}} \leq 1 / 2\right)$. Her total payoff will then be $4\left(1 p_{\mathrm{cl}}\right) \geq 2$ while the payoff of her neighbors will be $4 p_{\mathrm{cl}} \leq 2$. In consequence, this strategy will spread and give rise to a cross cluster (Fig. 2b). Payoffs in the next generation are

$\Pi_{a}=3 / 2+p_{\mathrm{cl}} \leq 2$

$\Pi_{c}=1 / 2+3\left(1 \quad p_{\mathrm{cl}}\right) \geq 2$

$\Pi_{d}=1+2 p_{\mathrm{cl}} \leq 2$ 
Both players $a$ and $d$ imitate $c$ and the cluster with greater $p$ continues growing. These results show that a strategy closer to the mean field solution, $p=1 / 2$, is able to invade the population if noise is present. No coexistence is possible in this setting. The underlying reason is the intrinsic weakening of clusters when its members cannot take exactly the same $p$.

\subsection{Cluster analysis: random roles $\{\mathrm{UI} / \mathrm{R} / \mathrm{nN}\}$}

When roles are randomly assigned, the same player can act twice as proposer or as responder when interacting with the same neighbor. As a result, stochasticity is introduced in the interaction between players of any type. The probability of acting twice as proposer, as well as twice as responder, is $1 / 4$, hence the expected payoff for a given interaction is

$$
E\left(\Delta \Pi_{i}\right)=\left(\frac{1}{4}\right) 2 \Delta \Pi_{i}^{O}+\left(\frac{1}{2}\right)\left[\Delta \Pi_{i}^{O}+\Delta \Pi_{i}^{R}\right]+\left(\frac{1}{4}\right) 2 \Delta \Pi_{i}^{R}=\Delta \Pi_{i}^{O}+\Delta \Pi_{i}^{R}
$$

which coincides with the case when roles are not random. In turn, the particular payoff increment for a concrete interaction can be quite different to this expectation, specially if the players are of different type (note, for instance, that if the player with lower $p$ acts twice as proposer the payoff increment for both players is zero). As a result, the boundaries of a cluster will fluctuate almost randomly. To study the effect of random roles the same cluster analysis than before will be used. Now we will pay attention to a stable cross cluster when it undergoes a boundary perturbation (Fig. 3). The subsequent evolution of the cluster will be computed by means of expected payoffs, so it can be seen as the average behavior of the perturbed cluster.

Let us consider first the case $p_{\mathrm{cl}}<p_{\mathrm{pop}} \leq 1 / 2$ (cluster further from $1 / 2$ than the rest of the population). Suppose a new individual is added at one edge of the cluster (position $a$ ). The payoff for a neighbor outside the cluster is $\Pi_{b}=3+\left(\begin{array}{ll}1 & p_{\text {pop }}\end{array}\right) \geq 3.5$, whilst for the neighbor inside $\Pi_{c}=2+2 p_{i} \leq 3$ (payoff for $a$ being lower than both). Thus, player $a$ imitates $d$ and the cluster reverts on average to the cross shape. On the other hand, if one of the arms of the cross is removed (position $a$ ) the updated payoff at that position will be $\Pi_{a}=3+\left(1 p_{\mathrm{pop}}\right) \geq 3.5$, whilst for the player previously in the middle of the cross $\Pi_{c}=3+p_{i} \leq 3.5$ and for the remaining arms $\Pi_{d}=1+3 p_{i} \leq 2.5$. As a consequence, players $c$ and $d$ will imitate its neighbors outside the cluster and the cluster will disappear.

In the opposite case, $p_{\text {pop }}<p_{\mathrm{cl}} \leq 1 / 2$. When the cluster is enlarged at one arm, the player at that position will get $\Pi_{a}=1+$ $3\left(\begin{array}{ll}1 & p_{\mathrm{cl}}\end{array}\right) \geq 2.5$, his neighbor outside the cluster $P i_{b}=3+p_{\mathrm{cl}} \leq 3.5$ and the neighbor inside $P i_{c}=2+2\left(\begin{array}{ll}1 & p_{\mathrm{cl}}\end{array}\right) \geq 3$. Provided that $p_{\mathrm{cl}}>1 / 3$ player $a$ imitates $b$ and the cross shape is recovered.
On the contrary, if an arm is removed (position $a$ ) payoffs will be

$\Pi_{a}=\Pi_{b}=3+p_{\mathrm{cl}} \leq 3.5$

$\Pi_{c}=3+\left(1 \quad p_{\mathrm{cl}}\right) \geq 3.5$

$\Pi_{d}=1+3\left(1 \quad p_{\mathrm{cl}}\right) \geq 2.5$

Since players $a$ and $b$ have neighbors outside the cluster with payoff 4 , this T configuration proves to be stable. However, removal of any of the remaining elements by further perturbations would result in its extinction. Hence, the $\mathrm{T}$ cluster should be considered as metastable.

We have thus seen that a majority of players with strategy close to $p=1 / 2$ is able to invade small clusters of players with lower $p$. On the other hand, a small cluster of players closer to $p=1 / 2$ can hardly invade a population with lower $p$ and boundary perturbations will make it eventually disappear. In conclusion, coexistence is not the expected outcome when roles are randomly assigned. Moreover, metastability gives advantage to players with higher $p$ when small clusters of similar size interact. This explains why the most frequent (but not the only one) outcome is fixation of strategies close to $p=1 / 2$.

\subsection{Cluster analysis: proportional imitation $\{\mathrm{PI} / n R / n N\}$}

Finally, let us analyze the case in which the source of noise is the choice of the neighbor to copy. Under proportional imitation, a player can adopt the strategy of any of her neighbors with payoff greater than own. As a result, given a certain configuration several transitions will be possible with different probabilities. To make things easier, we will take a qualitative approach and consider only which configurations are reachable from a given one. Note that transitions which require a player to imitate the strategy of a neighbor with lower payoff are not permitted. Payoffs can be calculated for every configuration as before, and moreover they will be exact if neither noise nor random roles are considered.

The possible dynamics for a $3 \times 3$ cluster are shown in Fig. 4 , where thick dark arrows are used when there is only a unique permitted transition (evolution is deterministic in that cases). Starting with a cluster of players with $p_{\mathrm{cl}}<p_{\text {pop }} \leq 1 / 2$ the cluster will fluctuate between $3 \times 3$ and cross configuration, but even tually will reach $\mathrm{T}$ configuration and subsequent extinction. On the other hand, if $p_{\mathrm{pop}}<p_{\mathrm{cl}} \leq 1 / 2$ both extinction and spreading can occur.

In conclusion, when proportional imitation operates no coex istence in the long term will be observable. Fixation is prone to occur for strategies with $p$ close to $1 / 2$ since small clusters of players of this type can spread (with certain probability) into a population with lower $p$ but not the opposite.

\section{Random roles $\{U I / R / n N\}$}

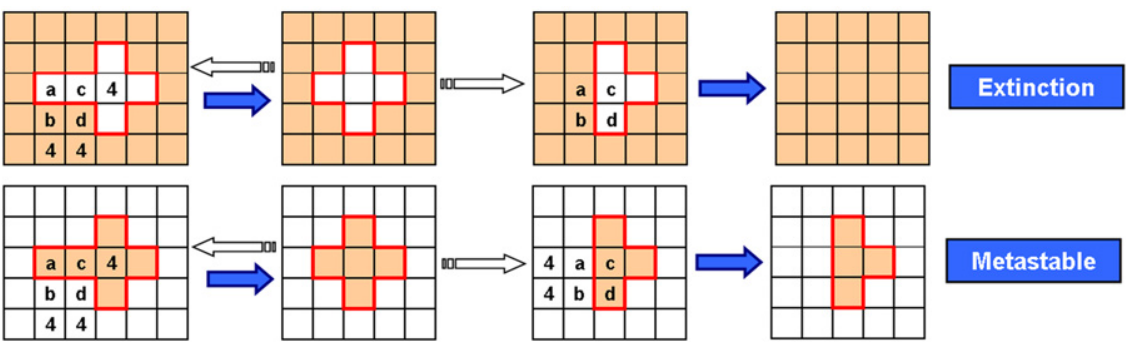

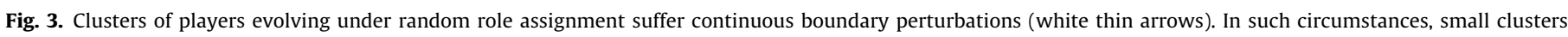

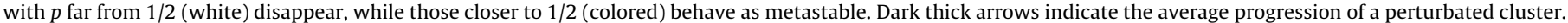
(For interpretation of the references to color in this figure legend, the reader is referred to the web version of this article.) 


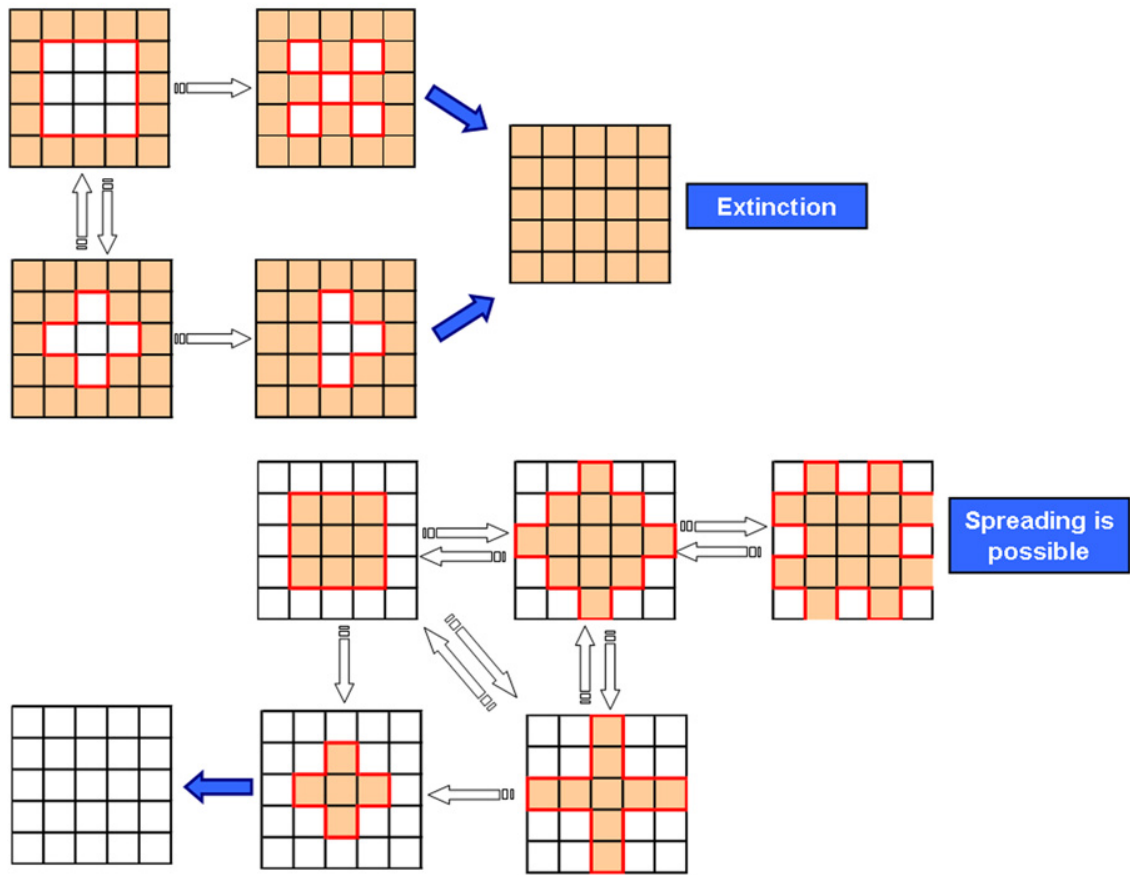

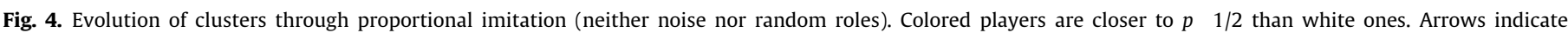

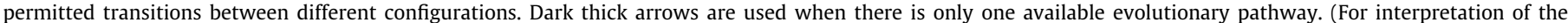
references to color in this figure legend, the reader is referred to the web version of this article.)

Table 2

Average final offers $(\bar{p})$ and standard deviations $(\sigma)$ in the empathetic case $p q$. High dispersion in setting $\{U I / n R / n N\}$ is due to stable coexistence of several strategies in the population. In all the other settings a unique final offer is reached in every realization. See text for an explanation of high dispersion in setting $\{U I / R / n N\}$ size $40 \times 40$.

\begin{tabular}{llllllll}
\hline Setting & Size & $\bar{p}$ & $\sigma$ & Setting & Size & $\bar{p}$ & $\sigma$ \\
\hline$\{U I / n R / n N\}$ & $10 \times 10$ & 0.323 & 0.127 & $\{P I / n R / n N\}$ & $10 \times 10$ & 0.435 & 0.034 \\
& $40 \times 40$ & 0.323 & 0.127 & & $40 \times 40$ & 0.482 & 0.008 \\
$\{U I / n R / N\}$ & $10 \times 10$ & 0.500 & 0.004 & $\{\mathrm{PI} / \mathrm{nR} / \mathrm{N}\}$ & $10 \times 10$ & 0.500 & 0.001 \\
& $40 \times 40$ & 0.500 & 0.004 & & $40 \times 40$ & 0.500 & 0.001 \\
$\{U I / R / n N\}$ & $10 \times 10$ & 0.397 & 0.064 & $\{P I / R / n N\}$ & $10 \times 10$ & 0.434 & 0.035 \\
& $40 \times 40$ & 0.405 & 0.150 & & $40 \times 40$ & 0.482 & 0.008 \\
$\{U I / R / N\}$ & $10 \times 10$ & 0.500 & 0.004 & $\{P I / R / N\}$ & $10 \times 10$ & 0.500 & 0.005 \\
& $40 \times 40$ & 0.500 & 0.004 & & $40 \times 40$ & 0.500 & 0.005 \\
\hline
\end{tabular}

\subsection{Size and connectivity effects}

In order to shed further light on the spatial Ultimatum game, we have also studied the effect of the system size and the connectivity of the lattice. A summary of the results for simula tions with different settings and network sizes is given in Table 2 . Finite size effects can be observed in small networks without noise, while the results become size independent when noise is added. This is due to the depletion of individuals with high $p$ at early steps of the evolution: starting with a population with random $p$ 's the maximum mean payoff does not correspond to individuals with $p=1 / 2$ but those with $p=1 / 3$ (Sinatra et al., 2009). As a result, individuals with $p$ close to $1 / 2$ are usually removed in small populations and cannot be recovered if noise is absent. As one can expect, this effect becomes specially relevant as connectivity increases and the real payoffs approach the well mixed limit (for instance, mean final offer reduces from 0.397 to 0.321 when increasing connectivity from 4 to 8 neighbors, setting $\{U I / R / n N\})$. One exception to this rule would be the setting
$\{U I / n R / n N\}$. Since coexistence is allowed for this setting the final configuration gets fixed early in the evolution. As a result, the mean final offer is close to the best one at initial steps, $p \approx 1 / 3$.

Another peculiar feature dependent on size is observed for setting $\{U I / R / n N\}$ (Fig. 1c). If the system is large enough a fraction of realizations ends with the fixation of very low offer strategies. Alternatively, for small networks all final fixed offers are distributed around the mean. This phenomenon also manifests itself in the standard deviation of the final $p$ distribution (Table 2), which increases dramatically with system size. The reason for this behavior is not obvious, but we believe that it is related to the survival of low $p$ clusters at initial steps of the evolution, in certain places of a large network and under favorable initial conditions. As it was seen before for this setting, once clusters are big enough their dynamics become dominated by random fluctuations, what gives a chance for fixation of that low $p$ clusters.

\section{Results: independent thresholds}

In the second part of our simulation program, we study the spatial Ultimatum game when players can independently choose their offers and their acceptance thresholds. The rational game theory prediction for this situation is the following: Given that the responder always receives greater payoff by accepting a deal than by rejecting it, the acceptance threshold $q$ will tend to zero and all offers will be accepted. As a consequence, proposers will offer the smallest amount possible. In conclusion, game theory predicts that both parameters, $p$ and $q$, will tend to zero. This is indeed the observed outcome for the evolutionary Ultimatum game in a well mixed population (Page et al., 2000). In this section, we will see how this result changes on a spatial setting. As in the previous section for empathetic players, here we will explore the station ary outcomes of the evolution when different evolutionary rules are considered. For this purpose, the comparison with the much simpler case of empathetic players will give insight into the causes behind the observed differences. 


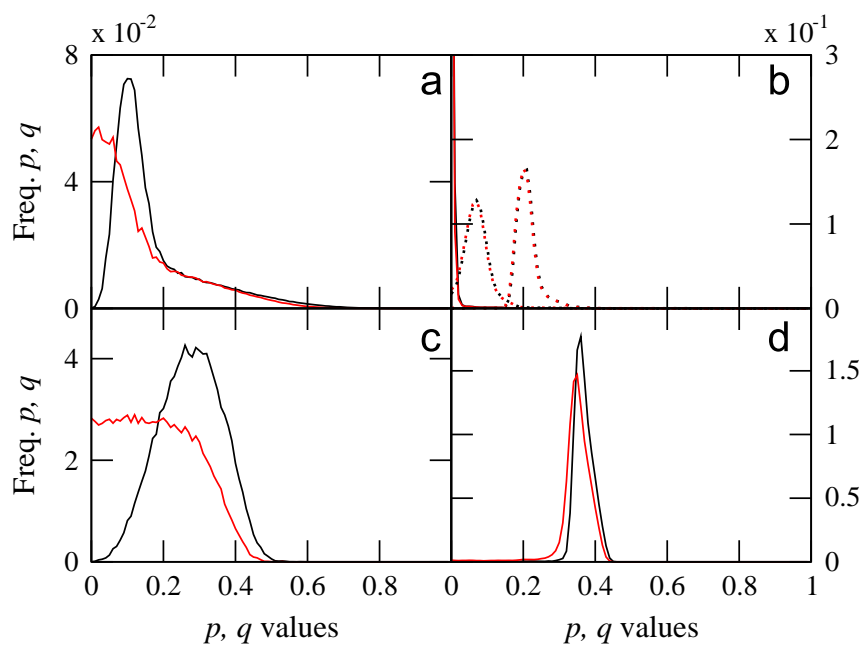

Fig. 5. Observed outcomes with independent $p$ and $q$. Black line corresponds to the distribution of offers $p$ and red line to the acceptance thresholds $q$, averaged for $10^{4}$ realizations. The four characteristic outcomes are (a) stable coexistence, only for the deterministic setting; (b) rational outcome, dotted lines represent distribution at intermediate time steps; (c) bell-shaped $p>0$ with $q$ close to zero - typical of small networks - and (d) "quasiempathetic" behavior, the most frequent outcome. (For interpretation of the references to color in this figure legend, the reader is referred to the web version of this article.)

\section{Table 3}

Average final offers $(\bar{p})$, acceptance thresholds $(\bar{q})$ and its standard deviations ( $\sigma_{p}$ and $\sigma_{q}$, respectively) for different settings and system sizes. High dispersion in setting $\{U I / n R / n N\}$ is due to stable coexistence of several strategies in the population. In all the other settings a unique final offer is reached in every realization. Quasiempathetic outcome in large networks produces similar values for $\bar{p}$ and $\bar{q}$ and small standard deviations, while rational outcome in $\{U I / n R / N\}$ leads to $\bar{p}$ and $\bar{q}$ close to zero.

\begin{tabular}{llllll}
\hline Setting & Size & $\bar{p}$ & $\sigma_{p}$ & $\bar{q}$ & $\sigma_{q}$ \\
\hline$\{U I / n R / n N\}$ & $10 \times 10$ & 0.280 & 0.127 & 0.146 & 0.129 \\
& $40 \times 40$ & 0.184 & 0.111 & 0.183 & 0.129 \\
$\{U I / n R / N\}$ & $10 \times 10$ & 0.012 & 0.029 & 0.011 & 0.023 \\
& $40 \times 40$ & 0.015 & 0.029 & 0.014 & 0.024 \\
$\{U I / R / n N\}$ & $10 \times 10$ & 0.285 & 0.087 & 0.189 & 0.112 \\
& $40 \times 40$ & 0.354 & 0.063 & 0.341 & 0.076 \\
$\{U I / R / N\}$ & $10 \times 10$ & 0.250 & 0.010 & 0.245 & 0.010 \\
& $40 \times 40$ & 0.336 & 0.009 & 0.330 & 0.010 \\
$\{P I / n R / n N\}$ & $10 \times 10$ & 0.319 & 0.080 & 0.222 & 0.119 \\
& $40 \times 40$ & 0.404 & 0.044 & 0.366 & 0.089 \\
$\{P I / n R / N\}$ & $10 \times 10$ & 0.306 & 0.081 & 0.219 & 0.117 \\
$\{P I / R / n N\}$ & $40 \times 40$ & 0.401 & 0.032 & 0.383 & 0.065 \\
& $10 \times 10$ & 0.318 & 0.081 & 0.223 & 0.118 \\
$\{P I / R / N\}$ & $40 \times 40$ & 0.405 & 0.043 & 0.363 & 0.093 \\
& $10 \times 10$ & 0.267 & 0.089 & 0.221 & 0.113 \\
& $40 \times 40$ & 0.377 & 0.028 & 0.357 & 0.050 \\
\hline
\end{tabular}

Variations on the evolutionary rules give four qualitatively different outcomes for the game. Representative distributions of parameters $p$ and $q$ are depicted in Fig. 5 where we have verified that the simulations have indeed converged to an asymptotically stationary state. A summary of the results for different settings and networks is collected in Table 3. We can classify the simula tion results in four different types:

Coexistence of offers: As in the empathetic case, coexistence of markedly different strategies is only observed in the setting $\{U I / n R / n N\}$ (Fig. 5a). In turn, any source of stochasticity in the evolution (random roles, proportional imitation, noise) leads to an homogeneous final population. Coexistence of offers depends on the formation of stable spatial configurations, and therefore it cannot be observed if the game takes place on random networks (even with homogeneous degree).
Rational ( $p, q \approx 0$ ): In a first stage, parameters $p$ and $q$ concen trate around well defined values far from zero. Afterwards, both evolve gradually towards zero (Fig. 5b). This behavior is only observed in the setting $\{U I / n R / N\}$, i.e. when noise is introduced into an otherwise deterministic setting. Proportional imitation and random role assignment prevent the population from reach ing the rational solution.

Bell shaped $p>0$ with $q$ close to zero: Offer parameter $p$ takes a bell shaped distribution with values greater than zero. On the other side, distribution of the acceptance threshold $q$ takes significant values at zero (Fig. 5c). That means that in some realizations the population fixes the rational value for $q$. This outcome is typical of small networks when either noise or random roles are absent (excluding the aforementioned settings with rational and coexistence outcomes). Specifically it can be observed in $10 \times 10$ systems with settings $\{U I / R / n N\},\{P I / R / n N\}$, $\{P I / n R / N\}$ and $\{P I / n R, n N\}$.

Bell shaped "quasiempathetic" ( $p \approx q>0)$ : The population takes $p$ and $q$ greater than zero, with $q$ just slightly below $p$ (Fig. 5d). The distance between $p$ and $q$ is typically on the order of 0.02 , so the final population resembles an empathetic one. This is the most frequent outcome, that appears in large networks $(40 \times 40)$ whenever proportional imitation or random roles are taken into account (with or without noise). It also appears in small networks if noise and random roles are combined (inde pendently of unconditional or proportional imitation).

\subsection{An analytical argument for the case of quasiempathetic behavior}

Among the four different types of behavior summarized above, the most common outcome is quasiempathetic behavior, namely the fixation of a strategy with $q$ very close to $p$ and both greater than zero. This being the farthest result from rational prediction, we believe it is worth to analyze it in detail. Our explanation of this behavior proceeds in two stages: Firstly, we will deal with the disappearance of rational players in the population after the first time steps. Subsequently, we will discuss the efficiency of a quasiempathetic strategy when evolution is driven by local interactions.

To begin with, we have observed in our simulations the remarkable fact that rational strategies ( $p$ and $q$ close to zero) are no more found in the population after the first time steps, despite they can be present in the starting random configuration. Disappearance of rational strategies takes place not only in small $10 \times 10$ networks but also in larger $40 \times 40$ ones. One simple reason for this phenomenon can be found by looking at the average payoff of a rational player in a well mixed population. Assume that the parameters of the players in the population are uniformly distributed in $[0,1]$, as the initial condition in our simulations. Since the expected payoff for a player with strategy $p=q=0$ is equal to the average $p$ in the population, we have that $\Pi_{(0,0)}=1 / 2$, which in turn coincides with the average payoff in the population. This means that there will be strategies better than the rational one and that it will be suppressed when individuals copy more successful ones. Note that if one considers the values for $p$ and $q$ uniformly distributed in $[0,1 / 2]$, the expected payoff for a rational player becomes $\Pi_{(0,0)}=1 / 4$, while the average payoff in the population remains equal to $1 / 2$. As a result, selective pressure against rational strategy is strong and it will disappear easily even in large populations.

Interestingly, a straightforward consequence of this phenom enon is that noise is necessary for a rational outcome to occur. This is just because only noise can recover individuals with low $p$ and $q$ later in the evolution. Since changes introduced by noise are 
very small, evolutionary conditions must be such that gradual evolution through small changes is possible. As we will see later, this is not the case if random roles or proportional imitation are introduced.

Having dealt with the early disappearance of rational players, we will now focus on explaining why quasiempathetic strategies are successful in the spatial UG. Our approach relies on the fact that, when spatial structure is present and connectivity is low, competi tion among players takes place at a local level. Accordingly, a successful player is one that defeats its neighbors, no matter how optimal his strategy is from a whole population point of view. Consider the following example: a player with $q=0$ will accept every offer of his neighbors, whose $p$ 's we will assume lower than $1 / 2$ and $q>0$. As a result, that player will obtain payoff $p$ in an interaction, while his neighbor will receive $1 p>p$. This gives a payoff advantage to the neighbor, so if the game took place only between these two players the rational strategy would be beaten. It is clear that this argument loses applicability when the number of neighbors increases and the final payoff becomes less and less dependent on the result of a single interaction, so that in the limit of a large, totally connected population the optimal strategy is indeed the rational one. Nevertheless, for the square lattices here consid ered, it can be assumed that local competition between pairs of players is relevant. Accordingly, a "one against one" approach should give a good approximation of the processes that drive evolution in these populations, and we will stick to it in what follows, taking back the issue of multiple neighbors afterwards.

Firstly, assume that $q \leq p \leq 1 / 2$ for all players in the popula tion. It is an observable fact that this condition becomes fulfilled very soon in the evolution. Let us focus on player $i$ and define the distances $a=1 / 2 p_{i}, b=p_{i} \quad q_{i}$ and $c=q_{i}$. Neighbor $j$ 's strategies can be classified according to the order relationships between their thresholds $p_{j}, q_{j}$ and those of player $i$ (see Fig. 6). We now want to find the optimal combination for $p_{i}$ and $q_{i}$ in order to achieve greater payoff than one's neighbor, provided that players lack any knowledge about their neighbors.

Player $i$ receives lower payoff than her neighbor $j$ in three cases: If both thresholds $p_{j}$ and $q_{j}$ are (1) greater than $p_{i}$ or (2) just between $p_{i}$ and $q_{i}$, or if (3) $p_{i}>p_{j}>q_{i}$ and $q_{j}<q_{i}$. Since in the beginning of the game thresholds are randomly assigned accord ing to a uniform distribution, it is reasonable to assume (at initial steps) that the probability of $p_{j}$ and $q_{j}$ belonging to a certain interval is proportional to the length of that interval. Taking into account the above definition of the intervals $a, b$ and $c$ together

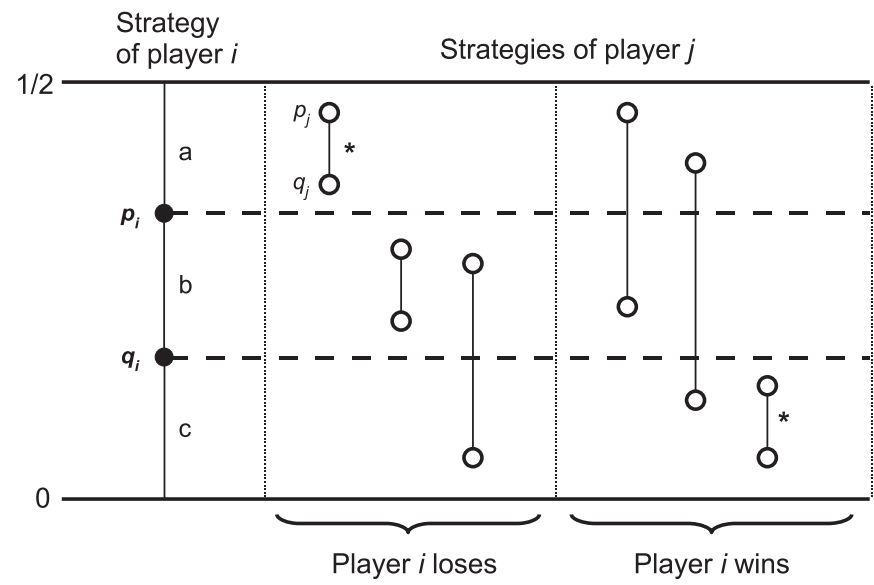

Fig. 6. Classification of the possible strategies of neighbor $j$ with respect to the thresholds of considered player $i$. Each strategy is represented by two open circles connected with a vertical line. Upper circle indicates the value of $p$ and lower circle that of $q$. Strategies marked with an asterisk are suboptimal, since only one deal per interaction is made. with the aforementioned constraint $q \leq p \leq 1 / 2$, the probability that player $i$ loses can be expressed as the function

$F(a, b, c)=4 a^{2}+4 b^{2}+8 b c$

On the other hand, player $i$ gets greater payoff than $j$ if (1) both thresholds of $j$ are below $q_{i}$, (2) $p_{j}>p_{i}$ and $p_{i}>q_{j}>q_{i}$, or if (3) $p_{j}>p_{i}$ and $q_{j} \leq q_{i}$. The same considerations as before for the probability of each combination give the following function for the probability that player $i$ beats his neighbor:

$G(a, b, c)=4 c^{2}+8 a b+8 a c$

The best strategy for $i$ must maximize $G(a, b, c)$ and minimize $F(a, b, c)$ subject to the constraints $a+b+c=1 / 2$ and $0 \leq a, b, c$ $\leq 1 / 2$. By writing $b=1 / 2 \quad a \quad c$, function $G$ becomes $G(a, c)=$ $4 a 8 a^{2}+4 c^{2}$, and it must be maximized in the triangle $0 \leq a \leq 1 / 2,0 \leq c \leq(1 / 2 a)$. The solution is $a=b=0, c=1 / 2$. In a similar fashion, it can be checked that function $F$ reaches its minimum for the same values. The conclusion of this analysis is that optimal strategy in a "one against one" game with the considered assumptions is $p=q=1 / 2$, what resembles the empa thetic outcome.

We must now discuss how this result changes if we recall that players interact with more than one neighbor and payoffs are the sum of all that interactions. From a phenomenological point of view, it means that player $i$ can also lose against neighbor $j$ if it performs very poorly in the interaction with the rest of his neighbors. Indeed, this is the case when $q_{i}$ is very high or $p_{i}$ is very low, what results in player $i$ making only one deal in every interaction. It is reasonable to assume that this kind of strategies are undesirable when multiple neighbors are considered, and players that make two deals in the interaction perform better than those leading to only one deal. Admittedly, in a quasiempa thetic situation, most individuals will make only one deal per interaction, but we believe that this effect will have less influence than using a strategy that from the very beginning would preclude one of the two exchanges (even for non quasiempa thetic players). Within this assumption, functions $F$ and $G$ are recalculated excluding the two strategies (marked with an aster isk in Fig. 6) for which only one deal is typically accepted. Thus these functions become

$F(a, b, c)=4 b^{2}+8 b c$

$G(a, b, c)=8 a b+8 a c$

The solution that maximizes $G$ and minimizes $F$ is $a=c=1 / 4, b=0$, that corresponds to strategy $p=q=1 / 4$, and once again, quasiem pathetic strategies are successful. We stress that we only have explicitly considered interaction between players $i$ and $j$ (local approach), while the effect of having more neighbors is included in the requisite for making typically two deals in every interac tion. For comparison with the well mixed setting, note that this requisite only precludes fixation of strategies that perform very poorly from a global point of view. Although it can seem very simplistic, this approximation is qualitatively consistent with experimental results and provides intuitive explanations to the exceptional observed outcomes. As a final note, observe that local analysis also predict early disappearance of rational strategies $(p=q=0)$, as we had already seen above in a well mixed context.

\subsection{Discussion of other outcomes}

As it has been just explained, two processes take place at the first steps in evolution that determine the fate of the whole population. First, strategies close to the rational one disappear, as a result of being less fit than the average. Second, quasiempa thetic strategies, with $q$ similar to $p$ and both located somewhere 
between $1 / 4$ and $1 / 2$, take advantage and spread through the population. This is indeed the final outcome in most of the cases, when no further evolution is possible. Nevertheless, differences appear in case unconditional imitation is chosen as the updat ing rule and roles are not randomly assigned. Finite size effects can also be found under certain circumstances and should be discussed as well.

Total lack of stochasticity (setting $\{U I / n R / n N\}$ ) results in coex istence of several strategies in the final population. As in the empathetic case, this is due to the formation of spatial clusters that can neither invade nor be invaded, and the same arguments that explained it before are valid here again. Perturbations induced by random role assignment and proportional imitation destroy spatial clusters and make coexistence inviable.

The only setting for which the rational outcome can be observed is $\{U I / n R / N\}$. This setting is the result of adding noise in the copy of parameters into an otherwise deterministic system. In fact, this is the only case in which evolution after fixation of a quasiempathetic behavior can occur. Firstly, it is straightforward to see that once a single strategy has been fixed, noise is necessary in order to let it evolve. Secondly, evolution of qua siempathetic strategies is not easy at all, since it requires a series of gradual processes that are very weakly selected for and, as a result, strongly sensitive to stochastic perturbations. Specifically, evolution from a homogeneous population towards the rational strategy requires the appearance of an individual with $q$ lower than the rest. This individual receives greater payoff than her neighbors and gives rise to a cross cluster (in average, provided that $p>1 / 5$ ). But the same noise that allowed this individual to appear produces variations inside the cluster that are of the same order of magnitude than the differences between the cluster and the population. As a result, there is not evolution as a cluster, but as individual players that successively appear. This slow, gradual evolution can take place in spatially ordered, almost deter ministic systems, but is suppressed as soon as stochastic pertur bations (induced by random roles or proportional imitation) are present. It can be therefore concluded that the quasiempathetic outcome is robust against noise, while the coexistence of strate gies resulting from deterministic setting $\{U I / n R / n N\}$ is unstable and evolves to the rational solution if imitation is not exact.

The last alternative outcome consists on a bell shaped dis tribution of offers greater than zero with acceptance thresholds close to zero. It only appears in small networks, where it is possible that no quasiempathetic individual exists at the begin ning of the simulation. Indeed, we have checked that if a small number of players with $p \approx q \approx 1 / 4$ are introduced in the starting configuration the standard quasiempathetic distribution is obtained as final outcome. Quasiempathetic outcome is also the natural one in small networks if noise and random roles are combined (settings $\{U I / R / N\}$ and $\{P I / R / N\}$ ). In those cases random role assignment induces fluctuations that result in slow fixation of strategies. This provides enough time so that noise generates quasiempathetic players.

Regarding the effect of connectivity, results do not suffer major changes when eight neighbors instead of four are considered in the game. Quasiempathetic behavior remains, although the mean final values for $p$ and $q$ decrease slightly $(\bar{p}=0.309, \bar{q}=0.284$ in setting $\{P I / R / N\})$. This result is in agreement with those observed in different networks (Page et al., 2000; Kuperman and Risau Gusman, 2008). At the same time, as connectivity increases a growing fraction of realizations ends with acceptance threshold close to zero. This deviation from quasiempathetic behavior is easy to explain, provided that higher connectivity reduces local competition (and therefore our local approach above ceases to hold). In any case, connectivity must be considerably higher than eight so that this effect becomes relevant.

\section{Conclusions}

We have studied in detail the Ultimatum game on a spatial setting, considering different update rules for the dynamics and introducing noise or randomness effects in several manners. Our simulations show that for a vast majority of settings (combinations of update rules, noise sources and one or two parameter strategies) evolution leads to the emergence of altruism, with offers and acceptance thresholds much larger than zero. Thus, empathetic players, who offer their own minimum acceptable amount, almost always evolve to fair offers, with values close to a 5050 split. The only situation in which this outcome does not arise is when the evolution is fully deterministic, i.e., under unconditional imitation with no noise. Furthermore, when the two parameters $p$ and $q$ are let to evolve independently, in most occasions they collapse to very close values for most cases, i.e., quasiempathetic behavior emerges spontaneously. This is a very interesting result because it resembles behaviors often found in experiments, above all regarding the offer (acceptance thresholds are in general significantly smaller). In this respect, let us note that a distribution of offers and thresholds very similar to the experimental ones was found for several combinations of dynamics (mostly for proportional imitation) and noise, but only for small systems. This suggests that the values observed in human populations might have arisen from evolution in small groups where competition is limited. On the other hand, the values we find for $p$ and $q$ in the quasiempathetic case are similar to those found by Page et al. (2000) for a different dynamics although, as we do not have data for the distribution of their values, we cannot take the comparison any further. Nevertheless, we believe that the dynamics in that previous work would most likely lead to similar distributions, in view of the role played by noise we have discussed above. In any event, the fact that the mean values are similar speaks in favor of the robustness of the result, in so far as it is only weakly dependent on the dynamics (it changes qualitatively only for special choices of update rules and noise).

Along the paper, we have been able to provide explanations for the simulation results using analytical arguments. Thus, for empathetic players we have shown that using the stability of square and cross clusters one can predict the final outcome of the evolution in the different dynamical scenarios. More importantly, we have also succeeded in developing an approximation to the case of two independent parameters for the strategies. Our results show that the convergence process can be understood in terms of a quick suppression of rational strategies followed, in a local (one or only a few neighbors) context, by the successful emergence of quasiempathetic players. Our approximation predicts that the offers should be between $1 / 4$ and $1 / 2$ of the total amount to share, values which are consistent with those reported in human experiments. This result agrees also with the finding that practi cally all dynamics lead to the emergence of a unique strategy throughout the population, coexistence being only possible in very few situations. While it is by no means the case that humans have all the same behavior in Ultimatum game experiments, we believe that the range of offers found through research all over the world is not that large as compared to the population. On the other hand, as was discussed in the Introduction, cultural effects could account for the coexistence of different values among cultures, while the individuals' response is very similar within monocultural groups (as is found, for instance, with European and US university students, whose range of variability is much smaller (Henrich et al., 2004).

We believe that the fact that quasiempathetic behavior is the generic outcome of the evolutionary, spatial Ultimatum game, is a relevant and non trivial one. Indeed, when empathy is imposed on the individuals from the beginning (by setting $p=q$ as a fixed condition), evolution generically leads to fairness with or without 
a spatial structure, as was shown by Page and Nowak (2002). Therefore, the spatial Ultimatum game does not add anything new. On the contrary, in the case in which the two parameters evolve independently, they first converge to similar levels because of a fast primary evolutionary process and, subsequently, quasiempathetic players retain offers much greater than zero as a consequence of a lack of later evolution. Indeed, if gradual evolution through small changes were effective, as it occurs for unconditional imitation with noise, the population will drift away from a fair split towards the rational solution with almost zero offers. Only this subtle two stage process leads to the emergence of fairness.

It is also important to put our conclusions in the context of findings on other networks. Our results are in contrast with those obtained by Kuperman and Risau Gusman (2008) on small world networks, as we observe fair behavior while they did not. We believe that the convergence to the rational behavior they observe is due to the destruction of the spatial structure (and hence of clusters) by the long range links. On the other hand, the results on complex networks reported by Sinatra et al. (2009) agree with the ones we are presenting here at least as far as the average values of offers and acceptance thresholds are concerned and for the case of propor tional imitation. In their system, however, acceptance thresholds tend to be not so close to offers, and therefore their agents are less quasiempathetic; in any event, the results are qualitatively similar, a remarkable conclusion in view of the differences among the net works studied in both works and of the influence that the type of network has on symmetric $2 \times 2$ games (Roca et al., 2009a,b).

To conclude, the picture that is beginning to emerge from our work and the previous ones is that the Ultimatum game on structured population is very robust under changes of the dynamics or the network of connections. The generic result is the appearance of fair behavior, with individuals that are quasiempathetic to some extent, and with little or no variability of strategies within the group. Therefore, as well mixed populations are hard to find in actual situations, and particularly so within humans, these results suggest that experiments should always show fair behavior because of the structure of the population, be it social or spatial. A word of caveat is in order here regarding the influence of the size of the groups as discussed in the text, that may lead to somewhat different out comes, including much lower acceptance thresholds. This must be taken into account when applying this kind of models to non human animals, particularly primates, where groups are not big and therefore effects as those described could be relevant. This conclu sion paves the way to further studies that try to combine biological evolutionary dynamics with cultural influences, as to provide a more complete picture of the emergence of fairness.

\section{Acknowledgments}

We are indebted to Susanna C. Manrubia for support and encouragement. We are very thankful to an anonymous reviewer and to José A. Cuesta for helping us finding the correct form of the analytical argument in Section 4.1. This work was supported in part by MICINN (Spain) through grants MOSAICO and RESINEE, and by Comunidad de Madrid (Spain) through grant MODELICO. J.I. is supported by a contract from the Consejería de Educación of the Comunidad de Madrid (Spain) and from the European Social Fund.

\section{References}

Axelrod, R., Hamilton, W.D., 1981. The evolution of cooperation. Science 211 1390-1396.

Boesch, C., Boesch, H., Vigilant, L., 2006. Cooperative hunting in chimpanzees. In: Kappeler, P.M., van Schaik, C.P. (Eds.), Cooperation in Primates and Humans. Springer-Verlag, Berlin, Heidelberg.

Brosnan, S.F., de Waal, F.B.M., 2003. Monkeys reject unequal pay. Nature 425, 297-299.

Burnham, T.C., 2007. High-testosterone men reject low Ultimatum game offers. Proc. R. Soc. London B 274, 2327-2330.

Camerer, C.F., 2003. Behavioral Game Theory. Princeton University Press, Princeton.

Eguíluz, V.M., Tessone, C.J., 2009. Critical behavior in an evolutionary Ultimatum game with social structure. Adv. Comp. Sys. 12, 221-232.

Fehr, E., Fischbacher, U., 2003. The nature of human altruism. Nature 425, 785-791.

Gintis, H., 2000. Strong reciprocity and human sociality. J. Theor. Biol. 206, 169-179.

Gintis, H., 2003. The Hitchhiker's guide to altruism: gene-culture coevolution and the internalization of norms. J. Theor. Biol. 220, 407-418.

Güth, W., Schmittberger, R., Schwarze, B., 1982. An experimental analysis of ultimate bargaining. J. Econ. Behav. Organ. 3, 367-388.

Hamilton, W.D., 1964. The genetical evolution of social behaviour I. J. Theor. Biol 7, 1-16.

Hammerstein, P. (Ed.), 2003. Genetic and Cultural Evolution of Cooperation (Dahlem Workshop Report 90). MIT Press, Cambridge, Massachusetts.

Hammerstein, P., 2006. Games and markets: economic behaviour in humans and other animals. In: Noë, R., van Hooff, J.A.R.A.M., Hammerstein, P. (Eds.) Economics in Nature: Social Dilemmas, Mate Choice and Biological Markets. Cambridge University Press.

Helbing, D., 1992. Interrelations between stochastic equations for systems with pair interactions. Physica A 181, 29-52.

Henrich, J., Boyd, R., Bowles, S., Camerer, C., Fehr, E., Gintis, H. (Eds.), 2004 Foundations of Human Sociality: Economic Experiments and Ethnographic Evidence from Fifteen Small-Scale Societies. Oxford University Press, Oxford.

Jensen, K., Call, J., Tomassello, M., 2007. Chimpanzees are rational maximizers in an Ultimatum game. Science $318,107-109$.

Kagel, J.H., Roth, A.E., 1995. The Handbook of Experimental Economics. Princeton University Press, Cambridge, Massachusetts.

Kappeler, P.M., van Schaik, C.P. (Eds.), 2006. Cooperation in Primates and Humans: Mechanisms and Evolution. Springer-Verlag, Berlin, Heidelberg.

Killingback, T., Studer, E., 2001. Spatial Ultimatum games, collaborations and the evolution of fairness. Proc. R. Soc. London B 268, 1797-1801.

Kuperman, M., Risau-Gusman, S., 2008. The effect of topology on the spatial Ultimatum game. Eur. Phys. J. B 62, 233-238.

Marlowe, F.W., et al., 2007. More 'altruistic' punishment in larger societies. Proc. R. Soc. London B 275, 587-592.

Maynard Smith, J., Szathmary, E., 1995. The Major Transitions in Evolution. Freeman, Oxford.

Milinski, M., 1984. Competitive resource sharing: an experimental test of a learning rule for ESSs. Anim. Behav. 32, 233-242.

Moran, P.A.P., 1962. The Statistical Processes of Evolutionary Theory. Clarendon Press, Oxford.

Nowak, M.A., 2006. Five rules for the evolution of cooperation. Science 314, 1560-1563.

Nowak, M.A., May, R.M., 1992. Evolutionary games and spatial chaos. Nature 359 $826-829$

Oosterbeek, H., Sloof, R., van de Kuilen, G., 2004. Cultural differences in Ultimatum game experiments: evidence from a meta-analysis. Exp. Econ. 7, 171-188.

Page, K., Nowak, M., 2000. A generalized adaptive dynamics framework can describe the evolutionary Ultimatum game. J. Theor. Biol. 209, 173-179.

Page, K., Nowak, M., 2002. Empathy leads to fairness. Bull. Math. Biol. 64, 1101-1116.

Page, K.M., Nowak, M.A., Sigmund, K., 2000. The spatial Ultimatum game. Proc. R. Soc. London B 267, 2177-2182.

Roca, C.P., Cuesta, J., Sánchez, A., 2009a. The effect of population structure on the evolution of cooperation. Phys. Rev. E 80, 46106

Roca, C.P., Cuesta, J., Sánchez, A., 2009b. Evolutionary game theory: temporal and spatial effects beyond replicator dynamics. Phys. Life Rev. 6, 208.

Sánchez, A., Cuesta, J.A., 2005. Altruism may arise from individual selection. J. Theor. Biol. 235, 233-240.

Schlag, K.H., 1998. Why imitate, and if so, how? A boundedly rational approach to multi-armed bandits. J. Econ. Theory 78, 130-156.

Sinatra, R., Iranzo, J., Gómez-Gardeñes, J., Floría, L.M., Latora, V., Moreno, Y., 2009. The Ultimatum game in complex networks. J. Stat. Mech. Theor. Appl., P09012.

Trivers, R.L., 1971. The evolution of reciprocal altruism. Q. Rev. Biol. 46, 35-57.

Wallace, B., Cesarini, D., Lichtenstein, P., Johanesson, M., 2007. Heritability of Ultimatum game responder behavior. Proc. Natl. Acad. Sci. USA 104 15631-15634. 